\title{
Variação Anatômica no Lenho de Astronium lecointei Ducke
}

\author{
Luiz Eduardo de Lima Melo¹, Cláudia de Jesus Silva², Cláudia Viana Urbinati, \\ Iêdo Souza Santos ${ }^{3}$, Warley Felício Soares ${ }^{2}$ \\ ${ }^{1}$ Universidade Federal de Lavras - UFLA, Lavras/MG, Brasil \\ ${ }^{2}$ Universidade do Estado do Pará - UEPA, Belém/PA, Brasil \\ ${ }^{3}$ Departamento de Tecnologia da Madeira - DTM, Universidade do Estado do Pará - UEPA, Belém/PA, Brasil
}

\begin{abstract}
RESUMO
Com o objetivo de avaliar a variação dos elementos celulares de Astronium lecointei Ducke no sentido medula-câmbio, analisaram-se amostras de madeira de três regiões radiais da árvore, a $1,30 \mathrm{~m}$ do solo: $0 \%$ (próximo à medula), 50\% (região intermediária) e 100\% (próximo ao câmbio vascular). A metodologia aplicada no trabalho foi aquela tradicionalmente recomendada para estudos em anatomia de madeira, seguindo orientação do IAWA Committee. Observou-se que alguns elementos anatômicos apresentaram tendências de variação definidas e estatisticamente significativas: frequência, diâmetro e comprimento dos elementos de vaso, altura dos raios em micrômetros e comprimento das fibras. Observou-se também maior obstrução dos poros por tilas próximo à medula e na região intermediária. A tendência de estabilização das dimensões dessas estruturas pode estar indicando a formação de madeira adulta a partir da região intermediária analisada no lenho da espécie.
\end{abstract}

Palavras-chave: Astronium lecointei, elementos celulares da madeira, variação radial.

\section{Anatomical Variation in the Wood of Astronium lecointei Ducke}

\begin{abstract}
In order to evaluate the variation of the cellular elements of Astronium lecointei Ducke from the pith towards the vascular cambium, wood samples from three regions of the radial sense in the tree at $1.30 \mathrm{~m}$ above the groundwere analyzed: $0 \%$ (near to the pith), $50 \%$ (intermediate region) and $100 \%$ (near to the vascular cambium). The methodology applied in the work was the one traditionally recommended for studies in wood anatomy, following the guidance of the IAWA Committee. It was possible to observe that some anatomical elements showed well-defined, statistically significant variation trends: frequency, diameter and length of the vessel elements, length of rays in microns, and fiber length. There was also greater pore blockage by tyloses close to the pith and in the intermediate region. The tendency to stabilize the values of these structures may indicate the formation of adult wood as from the intermediate region analyzed in the wood of Astronium lecointei Ducke.
\end{abstract}

Keywords: Astronium lecointei, cellular elements of wood, radial variation.

\section{INTRODUÇÃO}

A espécie Astronium lecointei Ducke - conhecida popularmente como muiracatiara, aroeira, muiracatiara-rajada, gonçalo-alves, aroeirão, dentre outros nomes, que variam de acordo com a região de ocorrência - pertence à família Anacardiaceae e encontra-se distribuída em toda a região amazônica, 
com maior frequência nos Estados do Pará e do Maranhão, onde habita principalmente as matas de terra firme (Loureiro et al., 2000; Lorenzi, 2002).

A árvore é de grande porte, com altura comercial que varia entre 15 e $20 \mathrm{~m}$, e sua madeira apresenta cerne de cor castanho-avermelhado, marcado por faixas de cor castanho-escuro em sentido vertical, de espaçamento variável, formando belas figuras que a distinguem da madeira de outras espécies. Por sua alta densidade, é muito comercializada na região e bem cotada tanto no mercado externo como no interno, sendo muito usada em produtos que requerem estabilidade dimensional, como móveis de luxo, pisos, carpintaria e marcenaria em geral (Loureiro et al., 2000).

O estudo da variação individual e a verificação da variabilidade que ocorre dentro da árvore, seja no sentido radial, seja no sentido axial, são importantes do ponto de vista da utilização tecnológica da madeira (Barrichelo \& Brito, 1979). O padrão de variação dos elementos celulares no xilema pode mudar muito, dependendo da espécie. Há, dessa forma, espécies que apresentam aumento na dimensão, na frequência, na proporção e no arranjo no sentido medula-câmbio ou base-topo, enquanto outras apresentam diminuição desses valores; há, ainda, aquelas espécies que praticamente não apresentam variação (Wilkes, 1988).

Segundo Zobel \& Talbert (1984), o padrão de variação dos elementos anatômicos pode estar relacionado à presença das madeiras juvenil, adulta e senescente no caule da árvore.

Para Ferreira et al. (2010), a ocorrência desses lenhos no mesmo fuste implica na existência de diferenças importantes nas propriedades das madeiras da medula até o câmbio, que podem ocasionar problemas no processo de industrialização da mesma, sendo, por isso, necessária uma prévia análise, a fim de aumentar o rendimento do processo.

Segundo Fan et al. (2009), os estudos que avaliam a variação radial e a variação axial dos elementos anatômicos no caule, além de possibilitarem um entendimento da arquitetura adotada pelas diferentes espécies para tornar eficiente e seguro o transporte de água, são de elevada importância comercial, pois, a partir dos resultados, é possível prever a matéria- prima mais indicada comercialmente para uma determinada aplicação.

Há uma série de estudos disponíveis que abordam as variações que acontecem no lenho e as suas influências sobre as propriedades da madeira em espécies de reflorestamento; entretanto, ainda são incipientes aqueles que se destinam ao estudo e à compreensão dessas variações no lenho de espécies de florestas nativas. Nestas, os indivíduos estão expostos às condições ambientais e geográficas específicas de cada habitat.

A partir do exposto, o objetivo deste trabalho foi estudar a variação da estrutura anatômica no sentido medula-câmbio na madeira de Astronium lecointei Ducke.

\section{MATERIAL E MÉTODOS}

O material avaliado nesta pesquisa foi cedido por uma serraria da Região Metropolitana de Belém-PA, que processa madeira proveniente do município de Gurupá, no Estado do Pará ( $01^{\circ} 24^{\prime} 18^{\prime}$ latitude Sul e $51^{\circ} 38^{\prime} 24^{\prime \prime}$ longitude oeste). O município de Gurupá apresenta clima tropical úmido, com temperatura média de $27^{\circ} \mathrm{C}$, tendo por época mais chuvosa os meses de janeiro a junho, com precipitação média anual em torno de $2.680 \mathrm{~mm}$, dominando o clima Af, na classificação de Köppen (IDESP, 1974).

As amostras foram retiradas a $1,30 \mathrm{~m}$ do solo (DAP), de três regiões do lenho, da medula em direção ao câmbio vascular: $0 \%$ (próxima à medula), $50 \%$ (intermediária) e 100\% (próximo ao câmbio).

De cada amostra, foram retirados corpos de prova com cerca de $1,5 \mathrm{~cm}^{3}$. Estes foram cozidos em água e glicerina na proporção de 4:1, até atingirem a condição ideal para o seccionamento. As secções histológicas foram obtidas em micrótomo de deslize (Leitz 1208), com espessura entre 15 e $18 \mu \mathrm{m}$, nos planos transversal e longitudinais tangencial e radial. Os cortes foram então clarificados em hipoclorito de sódio $60 \%$ e corados com safranina hidroalcoólica $1 \%$ (Johansen, 1940). Após a coloração e a desidratação dos cortes, foram montadas lâminas provisórias para mensuração dos elementos anatômicos, bem como lâminas permanentes em bálsamo do Canadá (diluído em acetato na proporção de 1:3), para documentação fotomicrográfica das estruturas 
anatômicas. Para a mensuração dos elementos anatômicos dissociados, foi preparado material macerado segundo o método de Franklin (1945). Avaliaram-se as características anatômicas conforme normas previstas pelo IAWA - International Association of Wood Anatomists Committee (IAWA, 1989). Realizou-se, para cada variável analisada (elementos de vasos - frequência/ $\mathrm{mm}^{2}$, comprimento e diâmetro; raios - altura e largura em micrômetros, frequência/mm linear; fibras - comprimento, espessura da parede e largura do lume), 25 contagens e medições.

As imagens microscópicas foram obtidas nos três planos de cortes usando um microscópio MOTIC ligado ao computador com ajuda do programa Aver tv.

O tratamento estatístico foi feito com o auxilio do programa Sistema de Análise de Variância - SISVAR. Realizou-se análise de variância e quando diferença significante foi detectada, aplicou-se o teste de Tukey para identificar os resultados determinantes das diferenças.

\section{RESULTADOS E DISCUSSÃO}

Na região da medula, foi constatada frequência média de vasos estaticamente semelhante à frequência da posição próxima ao câmbio, sendo estas superiores à posição intermediária (Tabela 1). Na região da medula, observaram-se vasos de menor diâmetro $(122,66 \mu \mathrm{m})$ e, nas posições intermediária e próxima ao câmbio, esses valores se estabilizaram (Tabela 1). Quanto ao comprimento dos elementos de vasos, observou-se que o valor médio encontrado nas posições próxima à medula e intermediária foi estatisticamente menor que o valor encontrado na posição próxima ao câmbio (Tabela 1).

Encontraram-se raios mais baixos na posição próxima à medula $(290,66 \mu \mathrm{m})$, havendo estabilização dos valores nas posições intermediária e próxima ao câmbio (Tabela 1). Para as outras características dos raios, não foram constatadas diferenças estatísticas.

Para características das fibras, a única variável que apresentou variação no sentido medula-câmbio foi o comprimento, o qual foi menor na posição próxima à medula $(780,66 \mu \mathrm{m})$, estabilizando-se nas posições posteriores (Tabela 1).

$\mathrm{Na}$ espécie analisada, observou-se que a frequência de vasos por $\mathrm{mm}^{2}$ apresentou-se oscilante da medula em direção ao câmbio, sendo que, na região próxima à medula e na próxima ao câmbio, essa variável apresentou maior frequência (Figura 1). Silva (1992) descreveu comportamento semelhante para os vasos da espécie Scoglottis guianensis Benth.,

Tabela 1. Variação radial das características anatômicas da madeira de Astronium lecointei Ducke.

Table 1. Radial variation of wood anatomical characteristics of Astronium Lecointei Ducke.

\begin{tabular}{|c|c|c|c|c|c|c|c|c|c|}
\hline \multirow{2}{*}{$\begin{array}{c}\text { Elementos } \\
\text { anatômicos }\end{array}$} & \multirow{2}{*}{$\begin{array}{c}\text { No de } \\
\text { contagens e } \\
\text { medições }\end{array}$} & \multirow{2}{*}{$\begin{array}{l}\text { Unidade de } \\
\text { medida }\end{array}$} & \multicolumn{3}{|c|}{ Posição radial } & \multirow[b]{2}{*}{ Min. } & \multirow[b]{2}{*}{ Max. } & \multirow[b]{2}{*}{$\mathbf{S}$} & \multirow{2}{*}{$\begin{array}{l}\text { CV } \\
(\%)\end{array}$} \\
\hline & & & $\begin{array}{c}0 \% \\
\text { (medula) }\end{array}$ & $\begin{array}{c}50 \% \\
\text { (intermediário) }\end{array}$ & $\begin{array}{c}100 \% \\
\text { (câmbio) }\end{array}$ & & & & \\
\hline \multicolumn{10}{|l|}{ Vasos } \\
\hline Frequência & 25 & $\mathrm{n} . / \mathrm{mm}^{2}$ & $10,8^{\mathrm{a}}$ & $8,80^{\mathrm{b}}$ & $11,53^{\mathrm{a}}$ & 5 & 23 & 2,44 & 24 \\
\hline Diâmetro & 25 & $\mu \mathrm{m}$ & $122,66^{\mathrm{b}}$ & $164^{\mathrm{a}}$ & $184^{\mathrm{a}}$ & 80 & 240 & 40,11 & 25 \\
\hline Comprimento & 25 & $\mu \mathrm{m}$ & $373,73^{b}$ & $419,33^{b}$ & $546^{\mathrm{a}}$ & 240 & 800 & 112,96 & 25 \\
\hline \multicolumn{10}{|l|}{ Raios } \\
\hline Frequência & 25 & $\mathrm{n} . / \mathrm{mm}$ linear & $6,8^{\mathrm{a}}$ & $7^{\mathrm{a}}$ & $8^{\mathrm{a}}$ & 4 & 11 & 1,36 & 19 \\
\hline Altura & 25 & $\mu \mathrm{m}$ & $290,66^{b}$ & $379,33^{\mathrm{a}}$ & $389,33^{a}$ & 180 & 520 & 77,71 & 22 \\
\hline Largura & 25 & $\mu \mathrm{m}$ & $43,33^{a}$ & $44^{\mathrm{a}}$ & $49^{a}$ & 10 & 70 & 12,38 & 27 \\
\hline \multicolumn{10}{|l|}{ Fibras } \\
\hline Comprimento & 25 & $\mu \mathrm{m}$ & $780,66^{\mathrm{b}}$ & $1056,66^{\mathrm{a}}$ & $1207,33^{a}$ & 430 & 1500 & 240 & 24 \\
\hline Largura do lume & 25 & $\mu \mathrm{m}$ & $9,24^{\mathrm{a}}$ & $7,88^{\mathrm{a}}$ & $8,17^{\mathrm{a}}$ & 5,32 & 12,6 & 1,71 & 20 \\
\hline $\begin{array}{l}\text { Espessura da } \\
\text { parede }\end{array}$ & 25 & $\mu \mathrm{m}$ & $3,05^{\mathrm{a}}$ & $3,12^{\mathrm{a}}$ & $3,39^{\mathrm{a}}$ & 1,4 & 4,20 & 0,64 & 20 \\
\hline Largura da fibra & 25 & $\mu \mathrm{m}$ & $15,37^{\mathrm{a}}$ & $14,11^{\mathrm{a}}$ & $14,94^{\mathrm{a}}$ & 10,1 & 21 & 2,26 & 15 \\
\hline
\end{tabular}

$\mathrm{Na}$ mesma linha, valores seguidos de letras distintas diferem estatisticamente entre si pelo teste de Tukey, considerando o valor nominal de 5\% de significância. Min. - mínimo; Max. - máximo; S - desvio padrão; CV (\%) - coeficiente de variação. 
ocorrente em mata alta de terra firme na Amazônia, na qual os vasos apresentam oscilação dos valores no sentido medula-câmbio, não mostrando um padrão definido de variação. Digby \& Wareing (1966) e Doley \& Leyton (1968) explicam que a frequência com que os elementos de vasos ocorrem em determinados estágios de desenvolvimento no lenho pode ser correlacionada com reguladores de crescimento, que apresentam grande influência na atividade cambial do vegetal. A alta concentração de
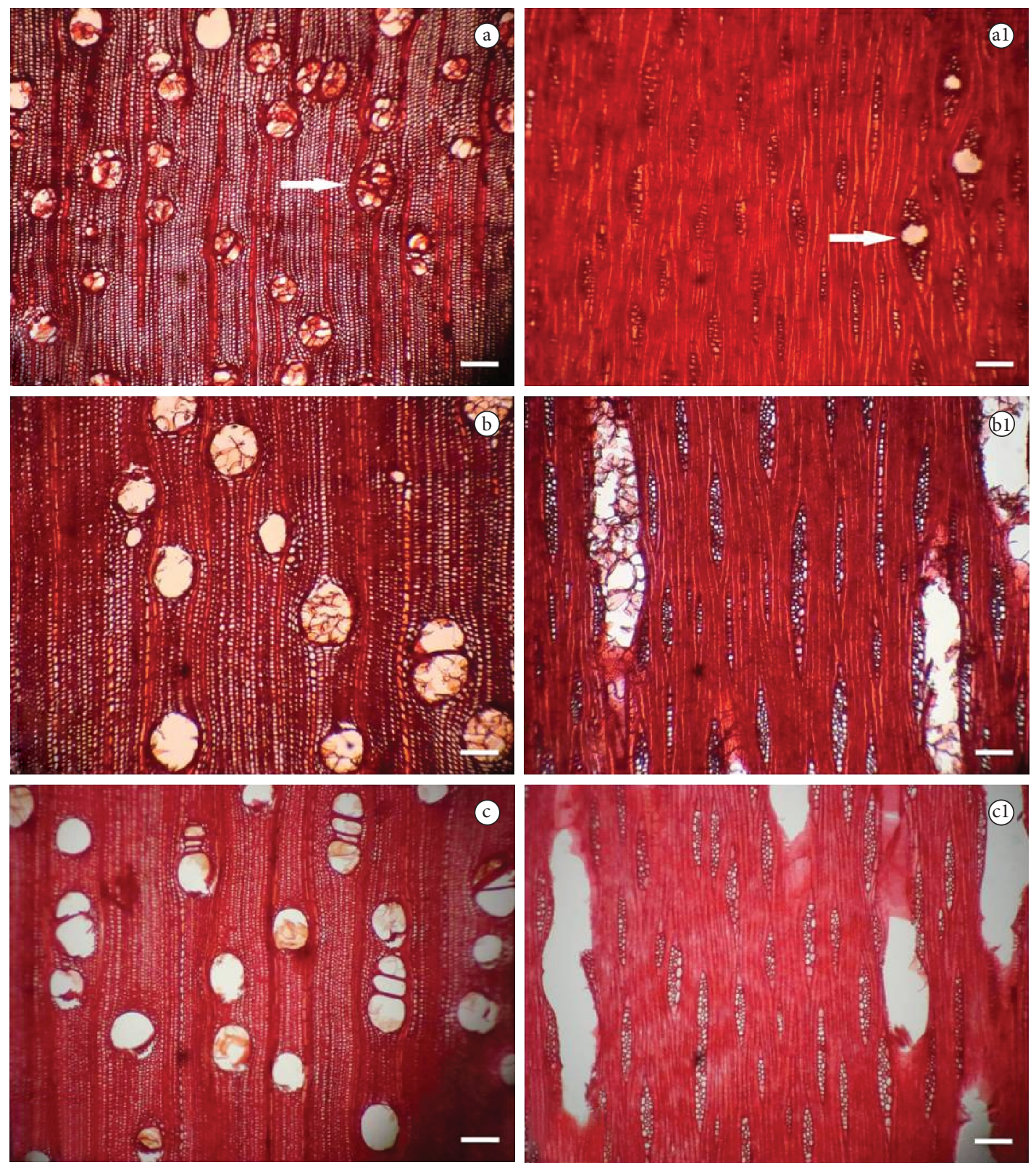

Figura 1. Variação radial em Astronium lecointei Ducke. a-c) Secções transversais. a) 0\% medula. Notar vasos obstruídos por tilos (seta). b) 50\% intermediária. c. 100\% câmbio. a1-c1) Secções tangenciais. a1) 0\% medula. Notar canais secretores radiais nos raios (seta). b1) 50\% intermediária. c1) câmbio. Observar diferença no diâmetro e frequência dos vasos e altura dos raios, da região da medula em direção ao câmbio. Barra $=170 \mu \mathrm{m}$.

Figure 1. Radial variation in Astronium lecointei Ducke. a-c) Transverse sections. a) 0\% pith (note blocked vessels by tyloses (arrow)). b) 50\% intermediate. c) 100\% cambium. a1-c1) Tangential sections. a1) 0\% pith (note radial secretory canals in the rays (arrow)). b1) 50\% intermediate. c1) cambium. Observe a difference in vessel diameter and frequency and height of the rays, the region of the spinal cord toward the exchange. Scale bars $=170 \mu \mathrm{m}$. 
auxina, por exemplo, produz madeira com grande quantidade de vasos, principalmente na sua fase juvenil, quando se tem intensa atividade fisiológica no vegetal (Zobel \& Talbert, 1984).

Já em relação ao comprimento dos elementos de vasos, verificou-se que a madeira estudada apresentou valores crescentes para esta estrutura no sentido medula-câmbio. Resultados semelhantes foram também descritos por Adamopoulos \& Vougaridis (2002), que observaram aumento no comprimento dos elementos de vasos em direção ao câmbio vascular para Robinia pseudoacacia. Testoni et al. (2009), para Plathymenia reticulata, e Lima et al. (2011), em estudos com a madeira de Cariniana legalis, verificaram o mesmo comportamento. Entretanto, Honjo et al. (2005), em estudo com Acacia mangium, não detectaram variação radial significativa para essa estrutura. Longui et al. (2011) observaram oscilação dos valores do comprimento dos vasos no sentido medula-câmbio para Pittosporum undulatum. Segundo Urbinati et al. (2003), os diferentes estudos realizados com elementos de vasos revelam que cada espécie, em função das suas características genéticas e das condições ambientais nas quais se desenvolve, apresenta padrões anatômicos diferentes, podendo ou não apresentar nitidamente os lenhos juvenil e adulto.

De forma geral, o aumento em comprimento dos elementos celulares que compõem o sistema axial do lenho, em função da idade, é resultado do aumento em comprimento das células que lhes originam, denominadas iniciais fusiformes. Sendo assim, os elementos de vasos, bem como as fibras, localizados nas camadas de crescimento mais próximas da medula e a um dado nível do tronco, apresentam menores dimensões do que aqueles localizados nas camadas finais de crescimento mais próximas ao câmbio (Tomazello Filho, 1987).

O diâmetro dos elementos de vasos também foi crescente no sentido medula-câmbio na espécie estudada. Essa tendência de variação foi também descrita por Tomazello Filho (1985, 1987), em estudos realizados com espécies de Eucalyptus sp.; Florsheim et al. (2009), para Eucalyptus dunnii; Fan et al. (2009), para quatro espécies de Fagaceaes, e Longui et al. (2009, 2010, 2011), analisando a variação radial do lenho de Luehea divaricata,
Piptadenia gonoacantha e Pittosporum undulatum, respectivamente. Para Fan et al. (2009), a variação crescente do diâmetro dos elementos de vasos no sentido medula-câmbio reflete o processo de maturação do câmbio vascular.

Observou-se que o diâmetro dos elementos de vasos de A. lecointei tendeu a se estabilizar nas duas posições externas do fuste, fato que pode estar relacionado à formação de madeira adulta na espécie (Figura 1). Para Tsuchiya e Furukawa (2009), o diâmetro dos vasos pode ser empregado como um indicativo para separar o lenho juvenil do adulto. Segundo Bendtsen e Senft (1986), a taxa de mudança na maioria das características anatômicas é muito rápida nos primeiros estágios de desenvolvimento do vegetal, mas posteriormente vão assumindo gradualmente as características da madeira adulta, havendo certo equilíbrio das dimensões e da frequência dos elementos celulares.

Para raios, há, de certa forma, poucos trabalhos que enfatizam a influência do crescimento em diâmetro da árvore sobre a morfologia dessa estrutura. Em relação à espécie estudada, o único parâmetro que apresentou tendência de variação definida foi a altura dos raios, sendo crescente da medula em direção ao câmbio e apresentando estabilidade do meio para a extremidade do fuste (Figura 1). Resultados semelhantes foram também observados por Giroud (1977), analisando a estrutura da madeira de Entandrophragma utile, Urbinati et al. (2003), analisando Terminalia ivorensis, e Longui et al. (2010), analisando Piptadenia gonoacantha.

Bannan (1956), em estudos realizados com Thuja occidentalis, verificou que a origem dos raios pode ser um processo altamente complicado, que envolve subdivisões transversais das iniciais fusiformes em várias células, perda de alguns produtos dessas divisões para a zona inicial e a transformação de outras em iniciais radiais. Esau (1974) explica que o aumento em altura dos raios ocorre por meio de divisões transversais de iniciais radiais completamente adultas e pela fusão de raios localizados um sobre o outro.

Quanto à variação radial das dimensões das fibras no sentido medula-câmbio para a espécie analisada, verificou-se que a única variável que apresentou diferenças estatísticas significativas entre 
as posições radiais avaliadas foi o comprimento das fibras, que apresentou tendência de variação crescente da medula em direção ao câmbio, com estabilização dos valores nas duas últimas posições avaliadas. $\mathrm{O}$ aumento no comprimento das fibras no sentido medula-câmbio foi também observado por Butterfield et al. (1993), Adamopoulos \& Vougaridis (2002), Honjo et al. (2005), Quilhó et al. (2006), Suckow et al. (2009) e Lima et al. (2011).

Gatto et al. (2008, 2010), em estudos com a madeira das espécies Luehea divaricata e Carya illinoinensis, respectivamente, observaram que o comprimento das fibras apresenta aumento significativo nos primeiros anos de vida, seguido de estabilização e decréscimo a partir de algumas camadas de crescimento mais próximas ao câmbio. Para os autores o comprimento das fibras foi um importante parâmetro para separar a madeira juvenil da adulta nas espécies analisadas.

Os vasos das posições próxima à medula e intermediária são quase completamente obstruídos por tilas e em uma proporção consideravelmente maior do que ocorre na posição próxima ao câmbio, caracterizando bem o cerne da espécie em estudo (Figura 1). Para Burger \& Richter (1991), a obstrução de vasos por tilas é atribuída a diferenças de pressão entre vasos e células parenquimáticas contíguas, pois, no período ativo de condução de fluidos nos vasos, a pressão dentro de ambas as células são mais ou menos constantes. Entretanto, à medida que ocorre a diminuição desse transporte no interior dos vasos, em virtude do crescimento em diâmetro da árvore e do início do processo de cernificação do lenho, a pressão no interior das células parenquimáticas torna-se maior, ocorrendo a expansão das mesmas para o interior dos vasos.

\section{CONCLUSÕES}

A estabilidade dos valores no diâmetro dos vasos, a altura dos raios em micrometros e o comprimento das fibras, nas posições intermediária e próxima ao câmbio vascular, podem ser considerados como um indicativo de formação de madeira adulta a partir dessa posição no lenho de Astronium lecointei Ducke.

Acredita-se que o padrão de variação radial diferenciado observado para a frequência de vasos por $\mathrm{mm}^{2}$ na espécie pode estar sendo influenciado por condições ambientais nas quais os indivíduos se encontravam. Contudo, para uma afirmação mais precisa sobre o assunto, faz-se necessária a realização de pesquisas que abordem a influência de fatores ecológicos sobre a atividade cambial da espécie.

\section{AGRADECIMENTOS}

Os autores agradecem à Xiloteca do Museu Paraense Emílio Goeldi, à Serraria HADEX Comércio e Indústria de Madeiras Ltda, e à Universidade do Estado do Pará, pelo apoio ao desenvolvimento deste trabalho.

\section{STATUS DA SUBMISSÃO}

Recebido: 31/03/2012

Aceito: 20/08/2012

Publicado: 28/02/2013

\section{AUTOR(ES) PARA CORRESPONDÊNCIA}

\section{Luiz Eduardo de Lima Melo}

Programa de Pós-graduação em Ciência e

Tecnologia da Madeira,

Departamento de Ciências Florestais, Universidade Federal de Lavras - UFLA, CEP 37200-000, Lavras, MG, Brasil e-mail: luizeduardo_47@yahoo.com.br

\section{Cláudia de Jesus Silva}

Universidade do Estado do Pará - UEPA, CEP 68745-000, Castanhal, PA, Brasil e-mail: claudia_jsilva@yahoo.com.br

\section{REFERÊNCIAS}

Adamopoulos S, Voulgaridis E. Within-tree variation in growth rate and cell dimensions in the wood of black locust (Robinia pseudoacacia L.). IAWA Jornal 2002; 23(2): 191-199.

Barrichelo LEG, Brito JO. Variabilidade radial da madeira de Pinus caribaea var. hondurensis. Instituto de Pesquisa e Estudo Florestal 1979; (18): 81-102.

Bannan MW. Some aspects of the elongation of fusiform cambial cells in Thuja occidentalis L. Canadian Journal of Botany 1956; 34(1): 175-196.

Bendtsen BA, Senft J. Mechanical and anatomical properties of individual growth rings of plantation- 
grown eastern cotton-wood and loblolly pine. Wood and Fiber Science 1986; 18(1): 23-38.

Burger LM, Richter HG. Anatomia da madeira. São Paulo: Nobel; 1991. 154 p.

Butterfield RP, Crook RP, Adans R, Morris R. Radial variation in wood specific gravity, fibre length and vessel area for two Central American hardwoods: Hyeronima alchorneoides and Vochysia guatemalensis: natural and plantation - grown trees. IAWA Journal 1993; 14: 153-161.

Digby J, Wereing PF. The relationship between endogenous hormone levels in the plant and seasonal aspects of cambial activity. Annals Botany 1966; 120: 607-622.

Doley D, Leyton L. Effects of growth regulating substances and water potential on the development of secondary xylem in Fraxinus. New Phytology 1968; 170: 579-594.

Esau K. Anatomia das plantas com sementes. São Paulo: Edgar Blucher; 1974. 284 p.

Fan X, Cao KF, Becker P. Axial and radial variations in xylem anatomy of angiosperm and conifer trees in Yunnan, China. IAWA Journal 2009; 30(1): 1-13.

Ferreira AL, Severo ETD, Calonego FW. Determinação das regiões dos lenhos juvenil e adulto pela mensuração do comprimento das fibras de Hevea brasiliensis. [cited 2010 nov. 6] Available from: http://prope.unesp. br/xxi_cic/27_22990637818.pdf.

Florsheim SMB, Couto HTZ, Lima IL, Longui EL. Variação nas dimensões dos elementos anatômicos da madeira de Eucalyptus dunnii aos sete anos de idade. Revista Instituto Florestal 2009; 21(1): 79-91.

Franklin GL. Preparation of thin sections of synthetic resins and wood - resin composites, and a new macerating method for wood. Nature 1945; 155(3924):51. http:// dx.doi.org/10.1038/155051a0

Gatto DA, Haselein CR, Buligon EA, Calegari L, Stangerlin DM, Oliveira LS et al. Estimativa de segregação do lenho de juvenil e adulto por meio de parâmetros anatômicos para a madeira de Luehea divaricata Mart por meio de parâmetros anatômicos da madeira. Ciência Florestal 2008; 18(4): 535-540.

Gatto DA, Haselein CR, Buligon EA, Calegari L, Stangerlin DM, Melo RR et al. Estimativa de segregação do lenho de juvenil e adulto por meio de parâmetros anatômicos para a madeira de Carya illinoinensis (Wangenh) K. Koch por meio de parâmetros anatômicos da madeira. Ciência Florestal 2010; 20(4): 675-682.

Giroud B. Statistical analysis of wood structure variation as related to distance from the pith in Entandrophragma utile (Meliaceae). IAWA Bulletin 1977; (4): 71-75.
Honjo K, Furukawa I, Sahri MH. Radial variation of fiber length increment in Acacia mangium Leinden. IAWA Journal 2005; 26(3): 339-352.

Instituto do Desenvolvimento Econômico-Social do Pará - IDESP. Estudo integrados da Ilha do Marajó. Belém; 1974. 333 p.

International Association of Wood Anatomists - IAWA COMMITTEE. List of microscope features for hardwood identification. IAWA Bulletin New Series 1989; (10): 226-332.

Johansen DA. Plant microtechnique. New York: McGraw-Hill; 1940. 523 p.

Lima IL, Lougui EL, Garcia MF, Zanatto ACS, Freitas MLM, Florsheim SMB. Variação radial da densidade básica e dimensões celulares da madeira de Cariniana legalis (Mart.) O. Kuntze em função da procedência. Cerne 2011; 17(4): 517-524.

Longui EL, Lima IL, Florsheim SMB, Bufolo A. Variação anatômica radial do lenho de açoita-cavalo (Luehea divaricata) e sua influência na densidade aparente. Revista do Instituto Florestal 2009; 21(2): 181-190.

Longui EL, Lima IL, Silva Junior G, Bufolo A, Suckow IMS, Florsheim SMB. Variação radial das características anatômicas, densidade aparente, teores de extrativos, lignina e holocelulose na madeira de Piptadenia gonoacantha (Mart.) Macbr . Instituto Florestal Série Registros 2010; 38(87): 341-353.

Longui EL, Romeiro D, Silva MT, Ribeiro A, Gouveia TCG, Lima IL, Florsheim SMB. Caracterização do lenho e variação radial de Pittosporum undulatum Vent. (pau-incenso). Hoehnea 2011; 38(1):37-50. http:// dx.doi.org/10.1590/S2236-89062011000100004

Loureiro AA, Silva MF, Alencar JC. Essências madeireiras da Amazônia. Manaus: MCT/INPACPPF; 2000. v. 4, 191 p.

Lorenzi H. Árvores brasileiras: manual de identificação e cultivo de plantas arbóreas nativas do Brasil. 4th ed. Nova Odessa: Instituto Plantarum; 2002. 382 p.

Quilhó T, Miranda I, Pereira H. Within-tree variation in wood fibre biometry and basic density of the urograndis eucalypt hybrid (Eucalyptus grandis x E. urophylla). IAWA Journal 2006; (27): 243-254.

Silva CA. Variação dimensional dos elementos xilemáticos em duas espécies madeireiras da Amazônia. Acta Amazonica 1992; 22: 261-274.

Suckow IMS, Longui EL, Lima IL, Florsheim SMB, Aguiar OT. Anatomia da madeira e densidade básica de angico-branco Anadenanthera colubrina (Vell.) Brenan. Instituto Florestal Série Registros 2009; 40: 131-135. 
Testoni LN, Longui EL, Lima IL, Florsheim SMB, Melo ACG. Anatomia, densidade aparente e velocidade de propagação do som na madeira de Plathymenia reticulata. Instituto Florestal Série Registros 2009; 40: 125-130.

Tomazello Filho M. Variação da densidade básica e da estrutura anatômica da madeira de Eucalyptus saligna e Eucalyptus grandis. Instituto de Pesquisa e Estudos Florestais 1985; 29: 37-45.

Tomazello Filho M. Variação da densidade básica e da estrutura anatômica da madeira de Eucalyptus globulus, Eucalyptus pellita e Eucalyptus acmeniodes. Instituto de Pesquisa e Estudos Flosrestais 1987; 36: 35-42.
Tsuchiya R, Furukawa I. Radial variation in the size of axial elements in relation to stem increment in Quercus serrata. Iawa Journal 2009; 30(1):15-26.

Urbinati CV, Azevedo AA, Silva EAM, Lisboa PLB. Variação estrutural quantitativa no lenho de Terminalia ivorensis A. Chev., Combretaceae. Acta Botânica Brasílica 2003; 17(3): 421-437. http://dx.doi. org/10.1590/S0102-33062003000300009

Wilkes J. Variations of wood anatomy within species of Eucalyptus. IAWA Bulletin 1988; 9: 13-23.

Zobel B, Talbert J. Applied forest tree improvement. John Wiley \& Sons, New York; 1984. 